\title{
Multiple Synchronous Tuberculous Aneurysms
}

\author{
Reuban D'cruz ${ }^{1}$ Dexter Y. S. Chan ${ }^{1}$ Pei Ho $\mathrm{Ho}^{2,3}$ \\ ${ }^{1}$ Department of Surgery, National University Hospital, Singapore \\ ${ }^{2}$ Department of Cardiac, Thoracic and Vascular Surgery, National \\ University Health System, Singapore \\ ${ }^{3}$ Department of Surgery, Yong Loo Lin School of Medicine, National \\ University of Singapore, Singapore
}

\author{
Address for correspondence Reuban D'cruz, MBChB, MRCS \\ (Eng), 5 Lower Kent Ridge Rd,Singapore 119074 \\ (e-mail: reuban.dcruz@gmail.com).
}

\begin{abstract}
The authors report a case of a 35-year-old woman with a medical history of recently diagnosed pulmonary tuberculosis presenting with chest and back discomfort. Chest radiograph demonstrated a bulky hilar mass, and computed tomography revealed multiple saccular aneurysms in the thoracic aorta, multiple fusiform aneurysms in the coronary arteries, left renal and splenic arteries, and right internal iliac pseudoaneurysms. Surgical management of the mycotic aneurysms was indicated in view of

Keywords

- tuberculous

- mycotic

- aneurysms symptomatology. Thoracic endovascular aortic repair (TEVAR) and ascending aortic replacement with aortic valve suspension and coil embolization of right iliac artery pseudoaneurysm were performed. The coronary artery aneurysms were managed with surveillance.
\end{abstract}

\section{Introduction}

Tuberculous aneurysms of the aorta and of the aortic arch are rare with a high risk of unpredictive rupture conferring life-threatening consequences. The first case of mycotic tuberculous aneurysms was reported in 1895 with sporadic case reports described since. To date, there have been only three cases of synchronous tubercular aneurysms in two different locations. ${ }^{1-3}$ Tuberculous mycotic aneurysms are typically seen in the descending thoracic aorta and abdominal aorta but are rarely noted in the ascending aorta or the iliac arteries. ${ }^{4}$ This is the first reported case of synchronous tuberculous mycotic aneurysms in seven different locations.

\section{Case Report}

A 35-year-old woman of Malay ethnicity with no reported medical history presented to our hospital with a 6-weeklong history of painless right supraclavicular lump and unintended weight loss. Cytology of the right supraclavicular lump demonstrated granulomatous necrotizing lymphadenitis, testing positive on polymerase chain reaction (PCR) for Mycobacterium tuberculosis. No organisms were detected on Gram staining of sputum. Sputum culture was sterile and was negative for acid-fast bacilli (AFB). Chest radiograph revealed a calcified nodule in the left lower lung lobe and a bulky left hilum. The patient was started on antitubercular therapy (ATT).

Computed tomography (CT) of the thorax was performed to characterize the bulky hilar mass, revealing multiple saccular aneurysms in the thoracic aorta with associated aortic root dilatation (-Figs. 1, 2). Multiple fusiform aneurysms were also noted in the left anterior descending coronary artery, right coronary artery, celiac trunk, and left renal and splenic arteries ( - Figs. 3,4 ). CT angiography of the aorta further revealed a right internal iliac artery aneurysm. Other radiologic findings include the presence of a calcified granuloma in the inferior lingular segment; a conglomerate of centrally necrotic nodes in the right supraclavicular nodes (-Fig. 5); and prominent nodes in the pre-carinal, para-aortic, retroperitoneal, and iliac regions. The patient's laboratory values were notable for elevated inflammatory markers (erythrocyte sedimentation rate $88 \mathrm{~mm} / \mathrm{h}$ and C-reactive protein $52 \mathrm{mg} / \mathrm{L}$ ). Bilateral carotid arteries were patent with peak systolic velocity less than $125 \mathrm{~cm} / \mathrm{s}$, and there were no intra- or extracranial aneurysms seen. Transthoracic echocardiogram revealed a dilated aortic root with aortic valve regurgitation.

Surgical intervention was discussed with the patient in view of symptomatic complaints of chest and back discomfort and impending risk of rupture. Informed consent was received

April 28, 2018

accepted after revision

July 9,2018

published online

November 27, 2018
DOI https://doi.org/

10.1055/s-0038-1675861

ISSN 2457-0214.
(C)2019 by Indian Society of

Vascular and Interventional Radiology
License terms

() (1) $\Theta \circledast$ 


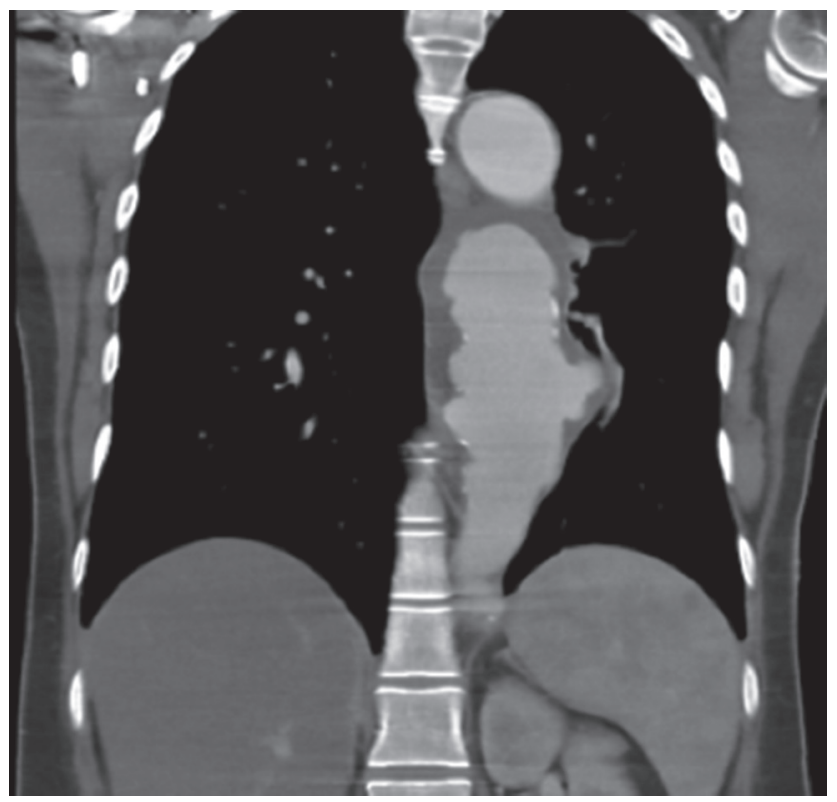

Fig. 1 Computed tomography demonstrating multiple saccular aneurysms along the descending thoracic aorta.

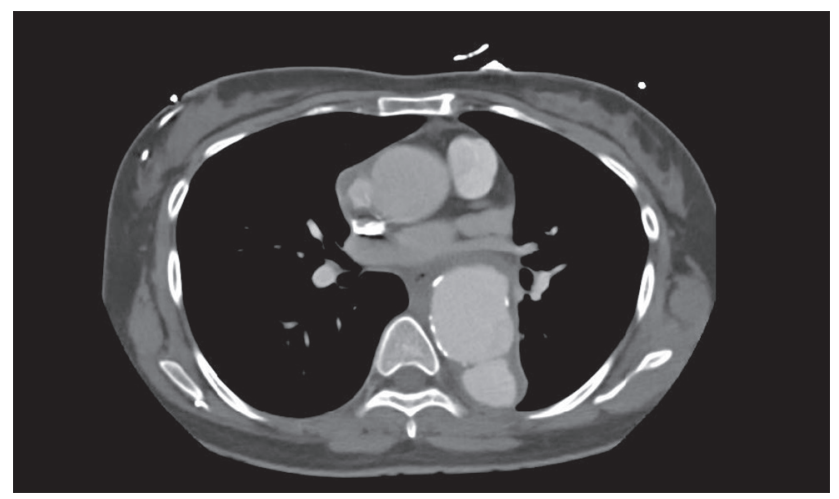

Fig. 2 Computed tomography demonstrating saccular aneurysm of the ascending aorta with aortic dilatation.

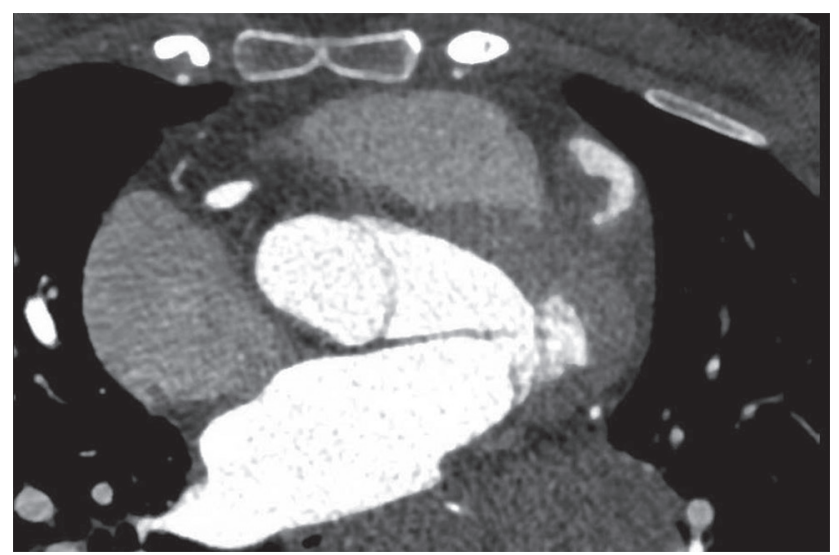

Fig. 3 Computed tomography demonstrating fusiform aneurysmal dilatation of the left anterior descending coronary artery.

obtained, and decision was made to proceed with thoracic endovascular aortic repair (TEVAR) and ascending aortic

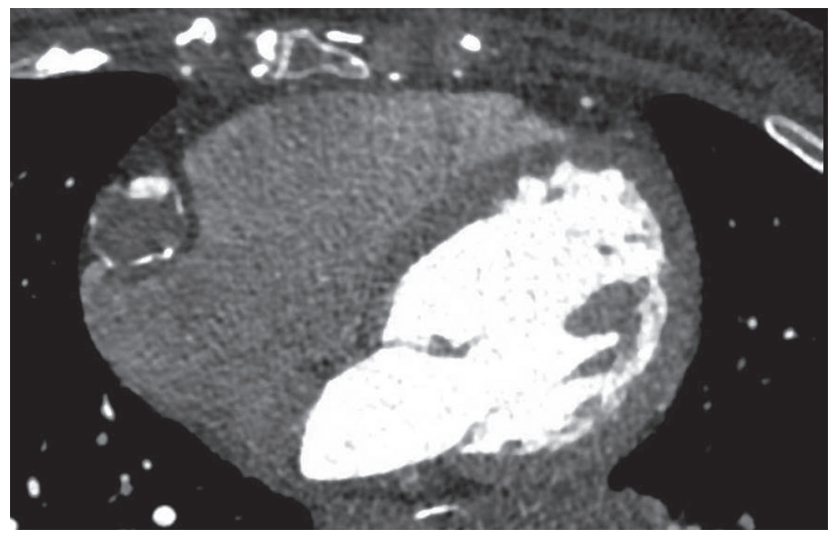

Fig. 4 Computed tomography demonstrating fusiform aneurysm of the right coronary artery.

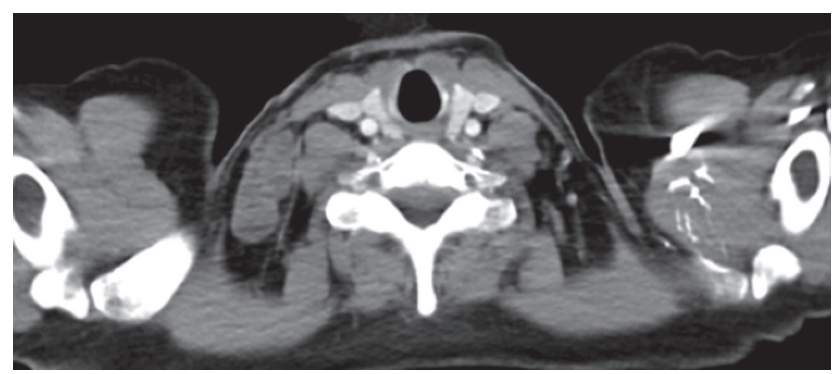

Fig. 5 Computed tomography demonstrating a conglomerate of right supraclavicular nodes.

replacement with aortic valve suspension. Infective pseudoaneurysm of the thoracoabdominal aorta was noted distal to the origin of the left subclavian artery extending proximal to the celiac trunk. Two Cook thoracic stent grafts (Cook Medical) were deployed, via left groin incision, proximal to the celiac artery origin and flush with the left subclavian artery origin. Molding between the two stent grafts and the distal landing zone was performed to reduce the risk of endoleaks (-Fig. 6). Post-TEVAR angiogram revealed preservation of flow into the left subclavian and celiac artery. Subsequently, coil embolization of the pseudoaneurysmal sac of the right internal iliac artery and its branches was performed. Postembolization angiogram revealed slow flow into the pseudoaneurysmal sac ( - Figs. 7, 8). Cerebrospinal fluid was drained preoperatively to protect the spinal cord.

Following a midline sternotomy, coronary bypass was performed via cannulations in the right atrium and distal ascending aorta. Ascending aorta was transected above the coronary ostium with the saccular pseudoaneurysm of the ascending aorta extending $4 \mathrm{~cm}$ distally. The adventitia of the aorta was also noted to be chronically inflamed. The ascending aorta and saccular aneurysm were resected, and a gel weave $28-\mathrm{mm}$ straight graft was used to reconstruct the ascending aorta. The patient was subsequently weaned off the bypass machine intraoperatively and discharged 10 days after surgery.

Conservative management was advocated for the coronary artery aneurysms with anticoagulation. Histologic examination confirmed that aneurysmal formation was tubercular in 


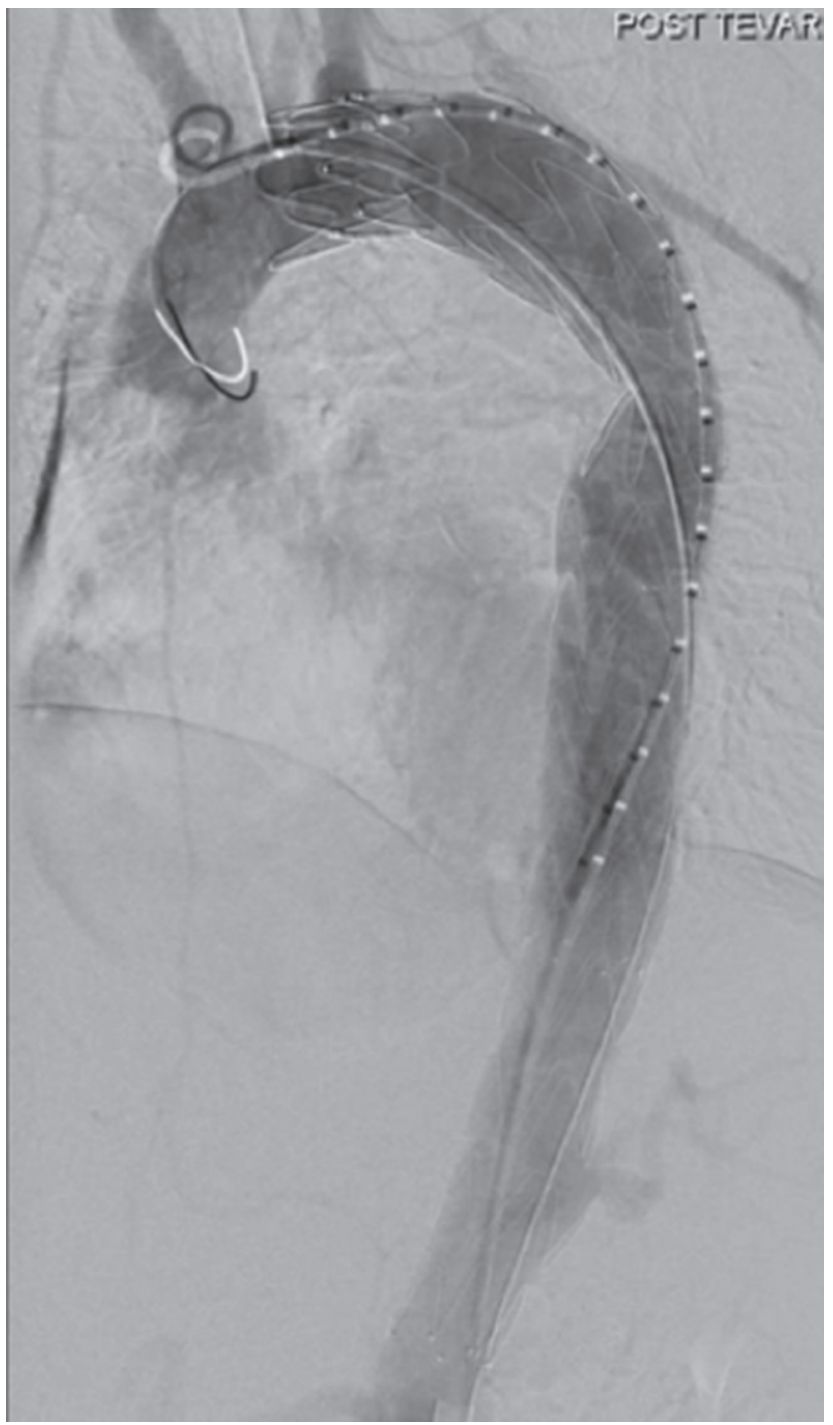

Fig. 6 Angiography after thoracic endovascular aortic repair (TEVAR).

origin. The patient completed 6 months of ATT and was started on anticoagulation when suitable. Stable aneurysms were noted in the coronary, renal, and splenic artery 12 months postoperatively. ( - Fig. 9).

\section{Discussion}

Tubercular mycotic aneurysms of the aorta and of the aortic arch are exceedingly rare entity. ${ }^{2}$ In a review of the medical literature by Long et al in 1999, only 41 cases have been recorded. Since then, there have been sporadic case reports of tubercular mycotic aneurysms varying in site of aneurysms. The aorta is the most frequent site of localization affecting the thoracic and abdominal aorta in equal ratios. ${ }^{4}$ Most of these aneurysms were saccular. ${ }^{4}$

The pathogenesis of vascular involvement in tuberculosis is not clearly understood. It is hypothesized that these aneurysms occur as a result of direct extension from a contiguous

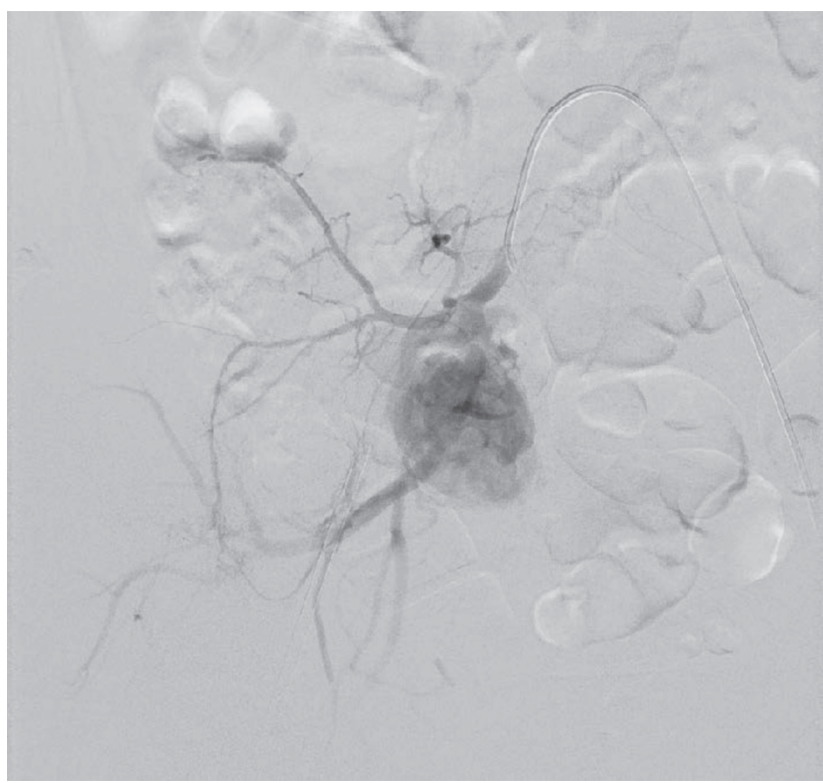

Fig. 7 Angiography of the right internal iliac artery aneurysm prior to embolization.

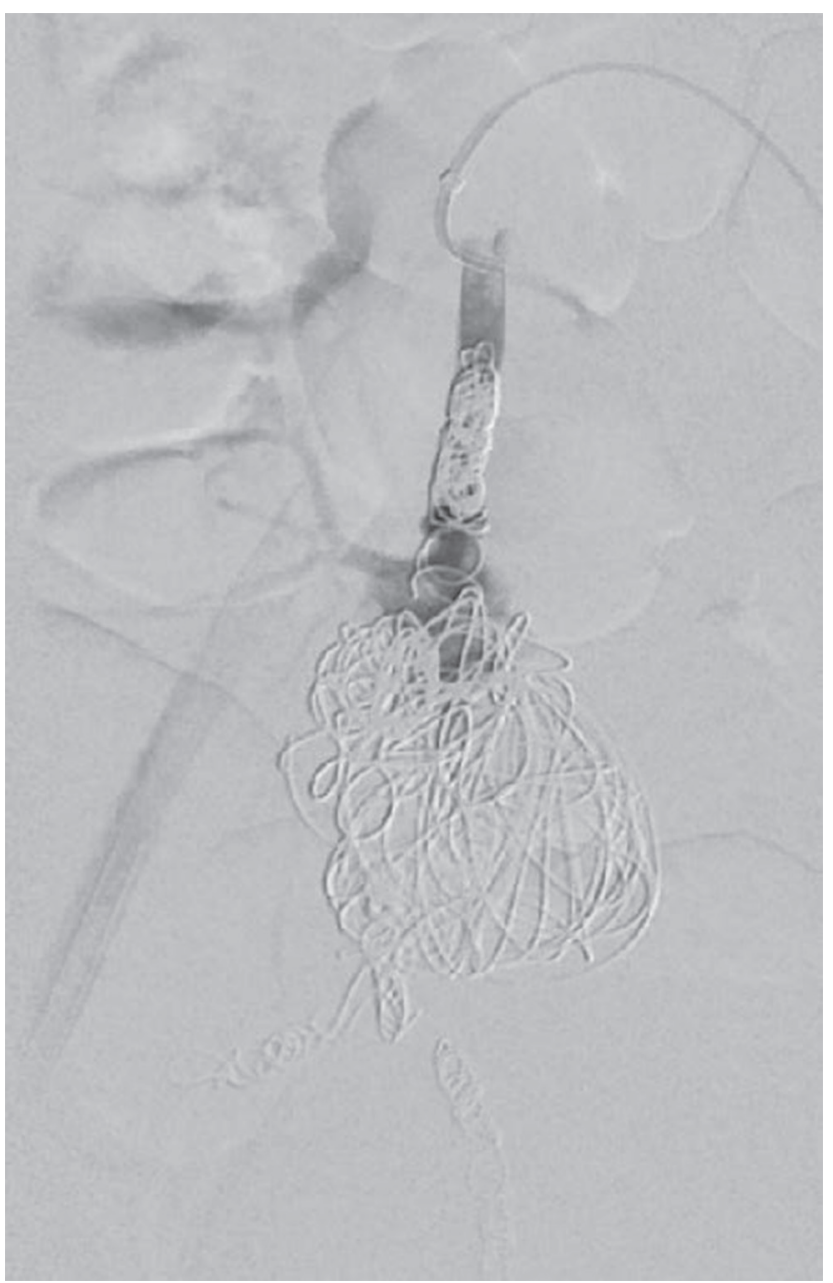

Fig. 8 Angiography of the right internal iliac artery aneurysm post coil embolization. 


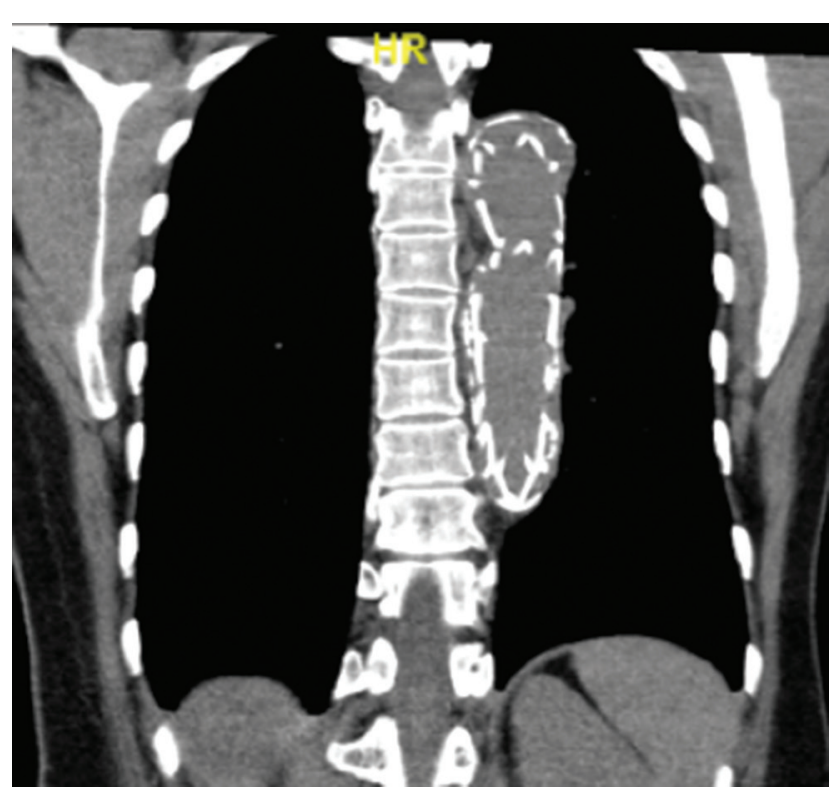

Fig. 9 Interval computed tomography 1 year after TEVAR.

tuberculous focus (usually pulmonary or lymphadenitis) or indirectly via lymphatics. ${ }^{5}$ When no primary contiguous focus was determined, hematogenous spread of the AFB with seeding in the adventitia and media via the vasa vasorum was the speculated mechanism. ${ }^{5}$ A primary focus of lymphadenitis of the right supraclavicular lymph node was identified in our patient with likely lymphatic spread of the pathogen to multiple sites of vascular system.

Clinically correlating the right supraclavicular mass with the likely unrelated chest and back discomfort was crucial in establishing the diagnosis. Surgical intervention was indicated in this patient for symptomatic presentation with radiologic concordance.

Computed tomography aortogram was crucial in delineating the type and extent of involvement the aneurysmal lesions, hence enabling for optimal preoperative planning. A transthoracic echocardiogram allowed for accurate description of the involvement of the aortic valve from the mycotic aneurysm. A coronary angiogram was not indicated where the risk of rupture outweighed the benefit of outlining these coronary aneurysms. Exclusion of mycotic infection of carotid, subclavian, intra-, and extracranial vessels allowed for less complex preoperative planning.

Combined surgical and medical management of tubercular mycotic aneurysm has accounted for the best outcomes. Long et al described 24 cases, with a mortality of $14 \%$, where surgical intervention and prolonged ATT were adopted. ${ }^{4}$ Hundred percent mortality was documented for the other 17 patients who opted for either surgery or ATT or neither options.

Traditionally, surgical intervention for mycotic aneurysms involves excision of infected tissues and vessel reconstruction via extra-anatomic bypass or in situ reconstruction. ${ }^{6,7}$ Despite risk of infection of vascular prosthetic graft, both methods of surgical management conferred similar and excellent results with adequate ATT. ${ }^{4}$ Canaud et al described favorable outcome in all three patients on follow-up (6 months, 8 years, and 10 years, respectively). ${ }^{5}$ The long-term durability of prosthetic grafts in the repair of tuberculous aortic aneurysms has yet to be clearly documented in the literature.

Since the advent of TEVAR, the efficacy of this surgical procedure in mycotic aneurysm has been explored. Previously advocated in patients at high surgical risk, there are infrequent case reports describing good outcomes for endovascular repair of these infective aneurysms. ${ }^{8}$ Han et al described complete resolution of pseudoaneurysm of the descending thoracic aorta with endovascular repair with adequate ATT at 1-year follow-up. ${ }^{8}$

Labrousse et al, however, expressed caution with endovascular repair, in view of the inability to excise and debride infective and necrotic material, thereby limiting the efficacy of ATT. ${ }^{9}$ This minimally invasive approach, therefore, confers a risk of recurrence of infection. They suggest that recurrence of disease in his patient had occurred shortly after discontinuation of 16-month-long ATT.

The main surgical management for infective ascending aorta aneurysm is an aortic replacement. ${ }^{10,11}$ Though there have been successful cases of primary patch repair of the aneurysmal lesion with no eventual false aneurysms seen on long-term follow-up, some authors feel that aortic replacement is the safer and better surgical option. ${ }^{10,11}$ Canaud et al argue that aortic replacement should be the surgical option of choice in view of difficulty in assessing the extent of aortic wall damage intraoperatively. ${ }^{5}$

There are no established guidelines for management of coronary artery ectasia (CAE), let alone those infective or inflammatory in etiology. ${ }^{12}$ Some authors advocate the use of antiplatelet or anticoagulation therapies in CAE due to the risk of thrombosis. ${ }^{13,14}$ There is an increased risk of myocardial infarction in inflammatory or infective CAE. ${ }^{13}$ Hence, prophylactic anticoagulation may be adopted as management of choice. Spontaneous rupture of CAE is rare, and the role of stenting has not proven to be beneficial. ${ }^{15}$

In branches of the aorta and in peripheral arteries where restoration of the arterial patency is not mandatory, coil embolization may be used together with medical therapy. ${ }^{5}$ As such, coil embolization was performed for the right iliac artery aneurysm in our patient.

In summary, multidisciplinary management of synchronous tuberculous aneurysms is imperative for the best clinical outcome.

\section{Conflicts of Interest \\ None.}

\section{Funding}

None.

\section{References}

1 Ringswald M, Roy TM. Synchronous mycotic aneurysms secondary to tuberculosis. J Ky Med Assoc 1989;87(7):320-324

2 Abaskaron M. Multiple pseudoaneurysms in a tuberculous patient. South Med J 1986;79(12):1582-1584

3 Mechchat A, Idrissi R, El Mahi O, et al. [Multiple tuberculous aortic aneurysms in a child. A case report] [in French]. J Mal Vasc 2008;33(4-5):218-220 
4 Long R, Guzman R, Greenberg H, Safneck J, Hershfield E. Tuberculous mycotic aneurysm of the aorta: review of published medical and surgical experience. Chest 1999;115(2):522-531

5 Canaud L, Marzelle J, Bassinet L, Carrié AS, Desgranges P, Becquemin JP. Tuberculous aneurysms of the abdominal aorta. J Vasc Surg 2008;48(4):1012-1016

6 Velayudhan BV, Idhrees AM, Sahu D, Jacob A. Open surgical repair of multiple tuberculous mycotic aneurysms of the thoracoabdominal aorta. J Thorac Cardiovasc Surg 2016;152(4):e95-e98

7 Choudhary SK, Bhan A, Talwar S, Goyal M, Sharma S, Venugopal P. Tubercular pseudoaneurysms of aorta. Ann Thorac Surg 2001;72(4):1239-1244

8 Han DK, Chung C, Walkup MH, Faries PL, Marin ML, Ellozy SH. Endovascular stent-graft repair of a tuberculous mycotic aortic aneurysm. Ann Vasc Surg 2011;25(5):699.e13-699.e16

9 Labrousse L, Montaudon M, Le Guyader A, Choukroun E, Laurent F, Deville C. Endovascular treatment of a tuberculous infected aneurysm of the descending thoracic aorta: a word of caution. J Vasc Surg 2007;46(4):786-788
10 Aebert H, Birnbaum DE. Tuberculous pseudoaneurysms of the aortic arch. J Thorac Cardiovasc Surg 2003;125(2):411-412

11 Bojar RM, Turner MT, Valdez S, Haskal R, McGowan K, Khabbaz KR. Homograft repair of a tuberculous pseudoaneurysm of the ascending aorta. Chest 1998;114(6):1774-1776

12 Devabhaktuni S, Mercedes A, Diep J, Ahsan C. Coronary artery ectasia-a review of current literature. Curr Cardiol Rev 2016;12(4):318-323

13 Dahhan A. Coronary artery ectasia in atherosclerotic coronary artery disease, inflammatory disorders, and sickle cell disease. Cardiovasc Ther 2015;33(2):79-88

14 Fukuhara S, Takase E, Fujimoto T, et al. [Coronary ectasia resulting in thrombotic coronary occlusion after warfarin interruption: a case report] [in Japanese] J Cardiol 2005;46(5):195-200

15 Kar S, Webel R. Diagnosis and treatment of spontaneous coronary artery pseudoaneurysm: rare anomaly with potentially significant clinical implications. Catheter Cardiovasc Interv 2017;90(4):589-597 